\title{
Maternal Psychological Control, Use of Supportive Parenting, and Childhood Depressive Symptoms
}

\section{BY}

Andrew L. Frazer

Submitted to the graduate degree program in Clinical Child Psychology and the Graduate Faculty of the University of Kansas in partial fulfillment of the requirements for the degree of Master of Arts.

Chairperson: Paula J. Fite, Ph.D.

Ric G. Steele, Ph.D., ABPP

Eric M. Vernberg, Ph.D., ABPP

Date Defended: March 2, 2015 
The Thesis Committee for Andrew L. Frazer

certifies that this is the approved version of the following thesis:

Maternal Psychological Control, Use of Supportive Parenting, and Childhood Depressive Symptoms

Chairperson: Paula J. Fite, Ph.D.

Date approved: March 9, 2015 


\begin{abstract}
Given the developmental importance of the family system, research on child depressive symptoms often examines the impact of parenting practices as either sources of or buffers against depressive symptoms. The current study, operating from a stress-process framework, examined the interactive effects of supportive parenting practices (i.e., mothers' use of positive communication, positive parenting, and parental involvement) and maternal psychological control on mother- and child-reported child depressive symptoms in a community-recruited sample of 9-12 year-olds. Discrepancies between mother- and child-reports of depressive symptoms were also examined. Maternal psychological control was uniquely associated with child-, not mother-, reported depressive symptoms. Parental involvement was uniquely associated with mother-, not child-, reported depressive symptoms. Positive parent-child communication was associated with less child- and mother-reported child depressive symptoms at the bivariate level, but not when unique associations were examined. Positive parenting was unrelated to either report of depressive symptoms. No interaction effects were detected. Implications and future directions are discussed.
\end{abstract}

Keywords: depression, parenting, stress-process, inconsistent parenting 


\section{Table of Contents}

Introduction.................................................................................

Supportive Parenting........................................................... 2

Psychological Control........................................................4

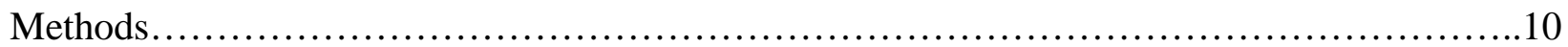

Participants....................................................................... 10

Measures...................................................................10

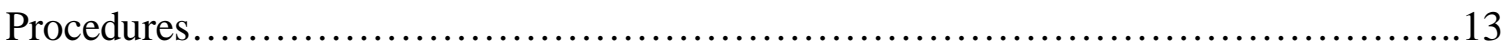

Data Analysis.................................................................

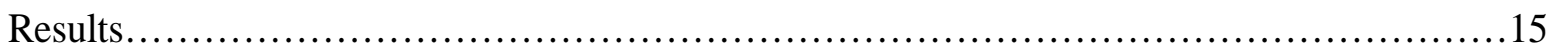

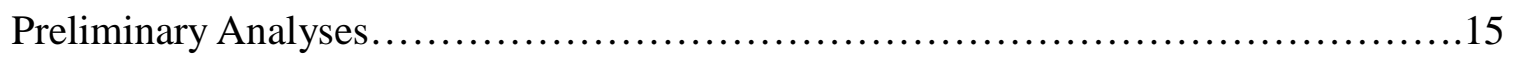

Regression Analyses........................................................... 16

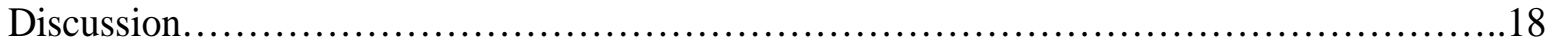

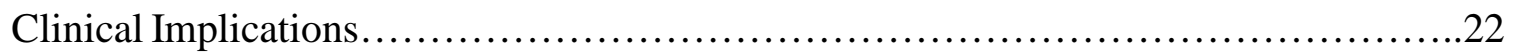

Limitations.............................................................. 23

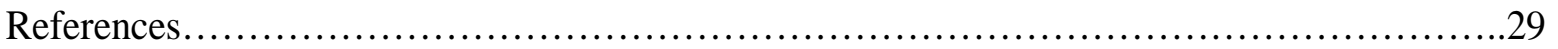

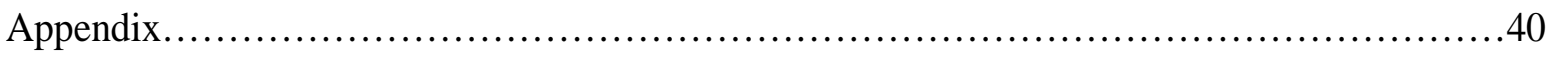




\section{Maternal Psychological Control, Use of Supportive Parenting, and Childhood Depressive Symptoms}

The onset of depression in children creates increased risk for current and lifetime psychosocial outcomes including substance abuse, academic problems, anxiety, suicide, eating disorders, and ODD (see Garber, Gallerani, \& Frankel, 2009). While rates of depressive disorders are generally lower in children than in adolescents (Garber et al., 2009; Lima, do Nascimento, de Carvalho, de Abreu, Neto, Brasil,...\& Reis, 2013), some estimate that as many as $9 \%$ of youth will experience at least one severe depressive episode by the time they reach the age of 14 (Lewinsohn, Hops, Roberts, Seeley, \& Andrews, 1993). Furthermore, the course of depression may in part be a function of how early symptoms emerge, such that early onset is associated with a chronic, episodic course of recurrent symptoms across a child’s lifetime (Costello, Pine, Hammen, March, Plotsky, Weissman, ...\& Leckman, 2002). Indeed, up to 84\% of individuals experiencing depression in childhood will experience subsequent depression in adulthood (Harrington, Rutter, \& Fombone, 1996). Therefore, given the prevalence of depressive symptoms reported in childhood, and the knowledge of the increased risk existing for individuals reporting these symptoms earlier in life, it behooves researchers to gain a more thorough understanding of the developmental pathways and contributing factors associated with this phenomenon. Doing so will aid in the implementation of early intervention and prevention efforts to ameliorate the risk factors for childhood depression.

Given the developmental importance of the parent-child relationship, research in the etiology of depression in children often begins at the family systems level, offering researchers the opportunity to examine the degree to which parents act as sources of or buffers against child depressive symptoms. When the influence of parenting on childhood depression is considered, 
two consistent themes emerge. Generally, high rates of harsh parenting and low rates of supportive parenting are associated with higher levels of child depressive symptoms (Dallaire, Pineda, Cole, Ciesla, Jacquez, LaGrange, \& Bruce, 2006; Wimsatt, Fite, Grassetti, \& Rathert, 2013). While much of the research on parenting and child development has aggregated parenting behaviors into typologies and styles, or considered the independent effect of individual parenting practices, relatively few studies have examined the interactive effect of specific parenting practices (Barber, Stolz, \& Olsen, 2005). Fewer still are the number of studies that examine the interactive effect of co-occurring positive and negative parenting practices in their relation to depressive symptoms in children. Understanding the ways that parenting practices interact is key for interventions, especially when considering the importance of positive parent-child relationships in interventions targeting children’s depressive symptoms (e.g., Lyneham \& Rapee, 2006).

The current study examined the effect of co-occurring parental support (i.e., mothers' use of parental involvement, positive communication, and positive parenting) and stress (i.e., psychological control) on mother- and child-report of childhood depressive symptomology in a community sample of 9-12 year-olds. By examining the interactive effect of these competing practices within the same parent, the current study extends the current literature on interactive models of parenting and depression in children. Furthermore, the comparison of multiple reports of depressive symptoms in the presence of competing sources of parental support and stress extends previous research on the parent-child relationship's impact on multi-informant discrepancies.

\section{Supportive Parenting}


Much of the research on parenting and depression has focused on the dimension of supportive parenting. Supportive parenting involves practices characterized as warm and responsive (Dallaire et al., 2006). Such parenting practices work to instill in a child a sense of personal value and establish the parents as a source of safety during distress (Cicchetti \& Toth, 1998). With regard to depressive symptoms, the use of supportive parenting practices has been repeatedly shown to be negatively associated with childhood depressive symptoms (Brennan, Le Brocque, \& Hammen, 2003; DeLay, Hafen, Cunha, Weber, \& Laursen, 2012; Muris, Meesters, Schouten, \& Hoge, 2004; Plunkett, Henry, Robinson, Behnke, \& Falcon Pedro, 2007; Vazsonyi \& Belliston, 2006). In particular, parental communication, involvement, and positive parenting have been found to be negatively associated with depressive symptoms (Yu, Clemens, Yang, Stanton, Deveaux, ... \& Harris, 2006; Colder, Lochman, \& Wells, 1997; Bruce, Cole, Dallaire, Jacquez, Pineda, \& LaGrange, 2006).

Positive parent-child communication has demonstrated a consistent link to promoting healthy development, and has been shown to be negatively associated with outcomes such as persistent delinquency (Stouthamer-Loeber, Loeber, Farrington, \& Wikstrom, 2002) and internalizing symptoms (Bayer, Sanson, \& Hemphill, 2006). Positive communication fosters openness and responsiveness to the child's needs and concerns, thereby bolstering the sense of self and buffering against depressive symptoms, such that higher rates of communication are associated with lower levels of child depressive symptoms (Bayer et al., 2006). Indeed, the inverse of this is also evident, such that depressed youth often report less open communication and greater difficulty in communicating with their parents (Yu et al., 2006).

The degree to which parents are actively involved in their child's life is also related to positive outcomes. Parental involvement has been linked to decreased risky sexual behavior 
(Pearson, Muller, \& Frisco, 2006), higher rates of academic achievement (Fan \& Chen, 2001), and social and behavioral success in school settings (El Nokali, Bachman, \& Votruba-Drzal, 2010). Most relevant to the current study, parental involvement is also negatively associated with anxiety and depressive symptoms (Colder et al., 1997; Duchesne \& Ratelle, 2010).

Positive parenting is a larger category of supportive parenting that encompasses behaviors characterized by warmth, acceptance, positive reinforcement, and expressions of praise and approval (Bruce et al., 2006). These behaviors serve to establish in the child a positive view of the self and of the world. The use of positive parenting practices has been linked to lower rates of externalizing behavior (Boeldt, Rhee, DiLalla, Mullineaux, Schulz-Heik, Corley, ... \& Hewitt, 2012; Healey, Flory, Miller, \& Halperin, 2011; Tolou-Shams, Paikoff, McKirnan, \& Holmbeck, 2007) in addition to lower rates of child depressive symptoms (Bruce et al., 2006; Compas, Champion, Forehand, Cole, Reeslund, Fear, ... \& Roberts, 2010; Gaté, Watkins, Simmons, Byrne, Schwartz, Whittle, ... \& Allen, 2013).

The aforementioned supportive practices are examples of how parents can embody the kind of support system shown to buffer against the possible threat of distressing experiences leading to depressive symptoms. Unfortunately, parents are equally apt to be the source of distressing experiences themselves. Thus, it is necessary to also examine parenting practices that serve as a risk factor for childhood depression.

\section{Psychological Control}

Schaefer (1965), in his taxonomy of parenting behaviors, differentiated traditional forms of behavioral control (i.e., setting curfews, etc.) from psychological methods of control. Schaefer emphasized that psychological methods of control were especially deleterious to one's ability to develop autonomy. Decades later, Barber (1996) revisited the construct and expanded upon its 
definition to include manipulation and exploitation of the parent-child bond (e.g., love withdrawal and guilt induction), negative affect-laden expressions and criticisms (e.g., disappointment and shame), and excessive personal control (e.g., possessiveness and protectiveness).

Recent research on psychological control has adopted Barber’s expanded definition, further differentiating the three major components of the construct: manipulation and coercion, intrusion into the personal domain, and disrespect (Barber, Xia, Olsen, McNeely, \& Bose, 2012). An additional marker of this kind of control includes being non-responsive to the child's emotional and psychological needs, thereby stifling the child’s independent expression and autonomy, making it difficult to be aware of a strong perception of the self (Barber 1996; 2012).

Not surprisingly, psychological control has been shown to be predictive of both internalizing (Taylor, Lopez, Budescu, \& McGill, 2012) and externalizing symptomology (Coln. Jordan, \& Mercer, 2012), including depression (Barber, 1996; Mandara \& Pikes, 2008; Plunkett et al., 2007; Soenens, Luyckz, Vansteenkiste, Duriez, \& Goossens, 2008), anxiety (Drake \& Ginsburg, 2012), academic achievement (Barber \& Harmon, 2002), delinquency (Bradford, Barber, Olsen, Erickson, Ward, \& Stolz, 2004), and aggression (Murray, Dwyer, Rubin, Knighton-Wisor, \& Booth-LaForce, 2013). Considering the feelings of guilt, self-responsibility, dependency, low self-esteem, and depressed affect that often characterize the experience of psychological control (Barber, 1996), researchers have largely explored its relation to anxiety and depression. Barber et al. (2012) proposed that the chief mechanism leading to such negative outcomes is the derogation of the child's self-definition, self-efficacy, and identity.

There is some evidence to support the notion that psychological control may have differential effects when considering aspects of the parent-child relationship. In a recent study, 
relationship quality was found to moderate the association between psychological control and aggression (Murray et al., 2013), such that maternal psychological control was predictive of higher rates of aggression when adolescents reported low-quality relationships with their father. Conversely, maternal psychological control was predictive of lower rates of aggression when adolescents reported high-quality relationships with their father. Thus, it is important to examine the interactive impact of psychological control in light of other parenting practices.

Consistent with Barber's notion that psychological control is detrimental to a child's selfdefinition, self-efficacy, and identity (Barber et al., 2012), stress-process theory may best explain the impact of competing parenting practices on child depressive symptoms. Stress-process theory asserts that life events and chronic strains are more likely to result in distress when they function to diminish the self (Pearlin, Menaghan, Lieberman, \& Mullan, 1981). This concept of the self is traditionally broken down into two dimensions: mastery, the extent to which an individual feels in control of the forces affecting his or her life, and self-esteem, the judgments one makes concerning self-worth. The effect of this damage to the self can be mitigated by successful coping behaviors as well as social supports, often (but not always) found through family and relational social networks.

Viewing the family context from a stress-process framework, parents are often studied as either sources of stress or social support. In most cases, however, families, and especially parents, are experienced subjectively as both sources of support and stress. In these cases, the impact of positive and negative parent behavior is not additive, but rather is interactive and integrative, such that individual parenting behaviors are appraised in the context of the overall parent-child relationship (Darling \& Steinberg, 1993; Lowe \& Dotterer, 2013). Indeed, several studies have examined the interactive effect of both positive and negative parenting practices (e.g., Dallaire et 
al., 2006; Eisenberg, Gershoff, Fabes, Shepard, Cumberland, Losoya, ... \& Murphy, 2001; Harper, Brown, Arias, \& Brody, 2006), suggesting that negative parenting behaviors (e.g., harsh discipline and negative emotional expression) may have the upper-hand when it comes to predicting future depressive symptoms. In a study by Dallaire and colleagues (2006), harsh, negative parenting was associated with greater depressive symptoms in children, regardless of the level of concurrent supportive positive parenting. Eisenberg and colleagues (2001) found similar results when considering the differential effect of parents' expression of positive and negative emotions.

Additionally, when the co-occurrence of supportive and unsupportive parenting behaviors happens concurrently, issues of inconsistency come into play. Children thrive under consistent parental behavior, discipline, and support, which have been shown to be predictive of positive adjustment (Bayer et al., 2006; Brennan et al., 2003). It is in this vein of thought that Turner and Finkelhor (1996) examined the effects of corporal punishment (i.e., a source of stress) and parental support co-occurring within the same parent. They found that children experiencing high levels of both support and corporal punishment experienced the greatest levels of distress. A study by Wimsatt and colleagues (2012) found similar findings when examining co-occurring high levels of parental communication and corporal punishment within the same parent. Through these studies, it would appear that experiencing a single parent as both a source of stress and support is particularly distressing, leading to worse outcomes. In such cases, a child can interpret inconsistent behaviors from a parent who is also a source of support (e.g., discipline) as a form of rejection (Rohner, Bourque, \& Elordi, 1996), thereby increasing confusion and distress.

\section{The Current Study}


Few studies examine the interactive effect of co-occurring positive and negative parenting practices in their relation to depressive symptoms in children. Fewer still are the numbe r of studies examining this co-occurrence within the same parent. Much of the extant research in this area examines the effect of each of these types of parenting practices separately, or classifies them under parenting typologies. By doing so, this inhibits examining their interactive effect. The current study contributes to the literature by examining the interactive effect of psychological control and three supportive parenting practices-mothers' use of positive parenting, parental involvement, and positive communication—on child depressive symptoms, utilizing a stress-process framework. Given that parents’ use of psychological control is an apparent source of stress (Barber, 1996), it follows that the concurrent experience of this parenting practice along with supportive parenting from the same parent (i.e., mothers) may operate similarly to the interactions in studies by Turner \& Finkelhor (1996) and Wimsatt et al. (2012). That is, the apparent inconsistency of experiencing these parenting practices at the same time may actually bring about greater distress for the child. While both supportive parenting practices and psychological control have each demonstrated a consistent link with depressive symptoms in previous research (Barber, 1996; Brennan, et al., 2003; DeLay et al., 2012; Muris et al., 2004; Plunkett et al., 2007; Vazsonyi \& Belliston, 2006), the current study is novel in its application of the stress-process model in the examination of both factors in their association with depressive symptoms in children.

The use of multiple informants in the current study is necessary when considering the nature of the constructs of interests. Psychological control is inherently dependent upon the child's perception; therefore using a child-report measure attains the most reasonable estimate of its occurrence. However, to account for the fact that depressed youth may often report their 
parents as overly negative or harsh (Garber et al., 2009), the use of parent-reported information was utilized to capture supportive parenting practices. With regard to the outcome measure, child depressive symptoms, the methodological difficulties and the alleged absence of a true "gold standard” (De Los Reyes \& Kazdin, 2005) associated with accurately assessing symptoms of psychopathology in children have made the use of multiple informants relatively commonplace. While children have been found to be adequate reporters of their own depressive symptoms (Kovacs, 1983), the examination of discrepancies between parent- and child-reports of depressive symptoms can yield clinically important findings. With regard to the current study, past research has demonstrated a link between discrepant parent- and child-reports of psychopathology and specific parenting practices (Kolko \& Kazdin, 1993; Treutler \& Epkins, 2003). Indeed, the degree to which parents and children endorse discrepant internalizing and externalizing symptomology has been shown to be related to parental acceptance and communication, such that higher levels of each are associated with less discrepant reports, and vice versa (Bidaut-Russel, Reich, Cottler, Robins, Compton, \& Mattison, 1995; De Los Reyes \& Kazdin, 2005; Kolko \& Kazdin, 1993; Treutler \& Epkins, 2003). Because the experience of concurrent stress and support from the same parent can be perceived as a form of rejection or non-acceptance (Rohner et al., 1996), it is likely that such circumstances will yield higher rates of child- than parent-reported child depressive symptoms.

Thus, three hypotheses guided the current study. First, all three supportive parenting practices were expected to be negatively associated with mother- and child-reported depressive symptoms. Second, psychological control was expected to be positively associated with motherand child-reported depressive symptoms. Next, psychological control was expected to interact with all three supportive parenting practices, such that high levels of psychological control in 
combination with high levels of supportive parenting would result in the highest levels of child-, but not mother-, reported child depressive symptoms, due to the inconsistent messages being relayed to the child.

\section{Methods}

\section{Participants}

Participants in this study were 76 children (53\% male) and mothers recruited from the community. Participants ranged from $9-12$ years of age $(M=10.38, S D=1.17)$. Researchers recruited participants for the study by placing flyers (see Appendix A) and advertisements in various community locations (e.g., sporting events, physicians’ offices, local restaurants, etc.). Parents and caregivers interested in participating in the study called the lab and were given a phone screen to ensure eligibility. To be eligible, families had to be English-speaking with children in the predetermined age range (9-12) at the time of the study. Furthermore, the presence of developmental delays, learning disabilities, or medication that would impede the child's ability to answer questionnaire items and interfere with reaction times precluded eligibility. Of the families who expressed interest in the study, 94 met criteria, though five of these families decided not to participate. Although the study targeted all caregivers, the majority of respondents were mothers (85\%). Thus, the current study focused only on mother caregivers.

Participants were representative of a mid-size Southeastern community sample. The majority of participants were Caucasian (72.4\%), with the remaining identifying as African American (21.1\%), Asian/Pacific Islander (1.3\%), or Biracial/Other (5.3\%). The median family income was approximately \$45,500 annually (\$0 - \$200,000). Less than one-third (31.6\%) of participating families received some form of public assistance.

\section{Measures}


Demographics. Parents and caregivers were asked to respond to a number of demographic items including age, gender, child's grade level, and measures of socioeconomic status (i.e., occupation, income, education level, and public assistance).

Positive Mother-Child Communication. Positive mother-child communication was measured using the Revised Parent Adolescent Communication Form (RPACF; Loeber, Farrington, Stouthamer-Loeber, \& Van Kammen, 1998). Mothers responded to 18 items addressing the degree to which they engage in positive and honest communication with their child. Items include questions such as "Can you discuss your beliefs with your child without feeling restrained or embarrassed?”; “Do you encourage your child to think about things himself/herself and talk about them so that they can establish their own opinion?”; or "Is your child free to say what he/she thinks in your family?” Response options ranged from " 1 - Never" to " 5 - Always." Responses were summed, such that higher scores indicated greater levels of positive communication. The RPACF has been utilized previously in numerous studies as a face valid assessment of healthy parent-child communication (Loeber et al., 1998; Loeber et al., 2000; Pardini, Fite, \& Burke, 2008). Internal consistency for this measure was acceptable ( $\alpha=.70)$.

Parental Involvement. The degree to which mothers demonstrate involvement in their child's life was assessed using six items from the Supervision/Involvement scale of the Pittsburgh Youth Study Questionnaire (PYSQ; Loeber et al., 2000). Mothers responded to items such as “Does your child help plan family activities?”; “Do you and your child do things together at home?”; or "Do you talk with your child about how he/she is doing in school?” on a scale ranging from " 1 - Never” to " 5 - Always.” Responses were summed, such that higher scores indicated greater levels of parental involvement. This measure of parental involvement has been utilized in previous research and demonstrates concurrent and predictive convergent validity 
with a variety of youth psychosocial outcomes such as aggression, delinquency, and substance use (Loeber et al., 1998). The internal consistency of this measure was acceptable $(\alpha=.77)$.

Positive Parenting. To assess Positive Parenting, mothers responded to the Positive Parenting Scale (Loeber et al., 1998). The Positive Parenting Scale includes eight items assessing reinforcement (e.g., "You reward your child when he/she does something good"), displaying affection (e.g., "You show your child affection when he/she does something good"), and verbal praise (e.g., "You say something nice about it when your child does something good"). Mothers rated the frequency of such practices on a range of " 1 - Never" through " 5 - Always." Responses were summed, such that higher scores indicate greater levels of parental involvement. Similar to the measure of parental involvement, this measure has been utilized in previous research and demonstrates concurrent and predictive convergent validity with a variety of youth psychosocial outcomes such as aggression, delinquency, and substance use (Loeber et al., 1998). The internal consistency of this measure was good $(\alpha=.84)$.

Psychological Control. Perceived parental use of psychological control was measured using the 10-item Psychological Control Scale (Barber, 1996). Prior to completing the measure, children reported whether they saw their parent on a regular basis (i.e., at least once a month). If not, these items were skipped. Children participating in the study responded to a total of 20 items on the PCS; two versions of ten identical items were given, one for each parent. Beginning each item with the prompt "My (Mother/Father) is a person who..." children rated behaviors such as “Often interrupts me,” "Blames me for other family members' problems,” and "Brings up my past mistakes when she/he criticizes me” on a scale of " 1 - Not like (her/him)," "2 - Somewhat like (her/him),” or “3 - A lot like (her/him).” Given the current study’s focus on mothers, only ratings of mothers' use of psychological control were utilized. Higher summed scores indicate 
greater levels of perceived psychological control. The PCS is an empirically validated measure (Barber, 1996) and frequently used assessment of perceived psychological control (Barber et al., 2012). Internal consistency for this measure was good ( $\alpha=.82)$ for reports of mothers.

Child-Reported Depressive Symptoms. Children responded to the Child Depression Inventory (CDI; Kovacs, 1983). The CDI is comprised of 27 items, each consisting of three separate statements describing depressive symptomology (e.g., how often the child feels like crying). Children respond by selecting the appropriate statement that describes their feelings in the past 6 months. The CDI is an empirically validated measure (Myers \& Winters, 2002) and frequently used screener for childhood depression (Kovacs, 1992; Nurcombe, Seifer, Scioli, Tramuntana, Lexington, \& Beauchesne, 1989). Responses were summed such that higher scores indicate greater experience of depressive symptoms. Internal consistency for this measure was $\operatorname{good}(\alpha=.86)$.

Mother-Reported Depressive Symptoms. Mothers' perception of their child's experience of depressive symptoms was assessed using the Withdrawn/Depressed scale of the Child Behavior Checklist (CBCL; Achenbach \& Rescorla, 2001). The Child Behavior Checklist is an empirically validated and widely used measure of emotion and behavior in children and assesses for both internalizing and externalizing symptoms of psychopathology (Achenbach \& Rescorla, 2001). The Withdrawn/Depressed scale consists of eight items characterizing social withdrawal and depressive symptoms. Internal consistency for this measure was acceptable ( $\alpha$ $=.74)$.

\section{Procedures}

All study procedures and materials were approved by the university’s Institutional Review Board. The data were collected as part of a larger study examining family, friends, 
school, and individual characteristics associated with aggression in children and included computerized tasks not relevant to the current study. Upon being invited to the lab, children and caregivers provided written assent and consent, respectively, to participate. Interviews with

children and caregivers took place separately and simultaneously. During each interview, trained study staff read each item of a series of questionnaires aloud and recorded respondents' answers on a computer using MediaLab software. Participants were assured confidentiality and were encouraged to be honest in their responses. Data collection sessions lasted approximately one and a half hours. Caregivers were compensated $\$ 45$ and children received a small prize for their time.

\section{Data Analysis}

Basic descriptive statistics (i.e., means, standard deviations, and ranges) were examined to establish the levels of positive communication, positive parenting, parental involvement, psychological control, and child depressive symptoms (both child- and parent- report) in the current sample. Skewness of study variables was also examined in order to determine whether the assumption of normality required for multiple linear regression was satisfied.

Correlation analyses were conducted in order to evaluate bivariate associations between variables, noting the strength and direction of any significant associations. Correlations between potential control variables (i.e., age, gender, and SES) and variables of interest were evaluated to determine which variables needed to be controlled for in the regression analyses. Consistent with Cohen’s (1988) criteria, r-values of .10, .30, and .50 were considered small, medium, and large effects, respectively.

Following this, multiple regression models were estimated in order to determine the moderating effect of supportive parent practices on the relation between psychological control 
and mother- and child-reported childhood depressive symptoms, examining interactive effects separately for power considerations. Child-reported child depressive symptoms were regressed onto positive communication, positive parenting, parental involvement, psychological control, and control variables in order to examine their first-order effects. Next, a multiplicative term between positive communication and psychological control was added to the model to evaluate an interactive effect. The same procedure followed for both positive parenting and parental involvement, evaluating a possible interaction between psychological control and these supportive parenting practices in their relation to child-reported child depressive symptoms. Subsequently, mother-reported child depressive symptoms were regressed onto each of the independent variables in a similar fashion. Patterns of results were considered across mother- and child-reports of child depressive symptoms. To aid in interpretation, all variables were standardized prior to regression analyses.

\section{Results}

\section{Preliminary Analyses}

Descriptive statistics were obtained for all study variables. Descriptive statistics (i.e., means, standard deviations, and skewness) are reported for study variables in Table 1. Values of

child reports of depressive symptoms were corrected for skewness $(\sqrt{y})$, but this did not significantly alter the pattern of overall findings. Thus, for ease in interpretation, the subsequent analyses are described using the original values.

To determine relationships among key study variables, bivariate correlational analyses were conducted. Results from these analyses are presented in Table 2. As anticipated, a moderate positive association was found between maternal psychological control and children's selfreported depressive symptoms, such that higher reports of psychological control were associated 
with greater number of child-reported depressive symptoms. Maternal psychological control was unrelated to mothers' ratings of their children's depressive symptoms.

Higher rates of positive parent-child communication were associated with lower rates of child depressive symptoms for both mother- and child-reports. A significant relationship was found between parental involvement and mothers' reports of child depressive symptoms, such that higher ratings of parental involvement were associated with lower rates of mother-reported child depressive symptoms. Conversely, parental involvement was unrelated to child-reported depressive symptoms. Interestingly, positive parenting was not associated with either children's nor mother's reports of depressive symptoms. Each of the three supportive parenting practices was significantly correlated with one another. Lastly, mothers and children's ratings of child depressive symptoms were unrelated to one another.

Only family income demonstrated a significant association with mothers' reports of child depressive symptoms, such that higher annual family income was associated with lower maternal reports of child depressive symptoms. Neither age, gender, nor ethnicity were found to be significantly related to child- or mother-reported child depressive symptoms. Thus, only family income was controlled for in subsequent regression models.

With regard to the outcome variables in the current study, child depressive symptoms, children's reports on the CDI indicated that a total of six participants (7.9\%) had elevated depressive symptoms (T scores $=56-69)$. By mothers' reports on the Child Behavior Checklist (CBCL; Achenbach \& Rescorla, 2001), six children fell within the Borderline Clinical range $(7.9 \%$; $\mathrm{T}$ scores $=65)$ and six children fell within the Clinical range for depressive symptoms $(7.9 \%$; $\mathrm{T}$ scores $=70-80)$.

Regression Analyses 
Child-Reported Depressive Symptoms. A series of multiple regression equations were then estimated to examine the interaction of maternal psychological control and the three supportive parenting practices in relation to children's report of child depressive symptoms. To determine first-order effects, children's reports of depressive symptoms were regressed onto maternal psychological control, positive communication, parental involvement, and positive parenting. Family income was also included as a control variable. See Table 3. None of the supportive parenting practices were uniquely associated with children's reports of their depressive symptoms. Only maternal psychological control was uniquely associated with childreported depressive symptoms.

Next, a multiplicative interaction term between maternal psychological control and positive parent-child communication was added to the model. No significant interaction was evident $(\beta=.09 ; \mathrm{t}=.68 ; \mathrm{p}=.50)$.

The same procedure of adding a multiplicative interaction term was utilized for examining psychological control as a moderator of parental involvement. Again, the interaction effect was not statistically significant $(\beta=-.00 ; \mathrm{t}=-.02 ; \mathrm{p}=.98)$.

A separate, third multiplicative interaction term between maternal psychological control and positive parenting was then added to the original first-order model, and once more no significant interaction effect was evident $(\beta=-.03 ; \mathrm{t}=-.27 ; \mathrm{p}=.79)$.

Mother-Reported Child Depressive Symptoms. To determine first-order effects, mothers' reports of their child's depressive symptoms were regressed onto maternal psychological control, positive communication, parental involvement, and positive parenting. As before, family income was included as a control variable (See Table 3). Diverging from the patterns observed with children's reports of depressive symptoms, maternal psychological control was unrelated to 
mothers' reports. Of the supportive parenting practices, only parental involvement was uniquely associated with mothers' reports of child depressive symptoms, such that mothers reporting greater involvement also reported lower child depressive symptoms.

Next, a multiplicative interaction term between maternal psychological control and positive parent-child communication was added to the model. No significant interaction was evident $(\beta=.01 ; \mathrm{t}=.06 ; \mathrm{p}=.96)$.

The interaction term between maternal psychological control and parental involvement was then added to the previously estimated first-order model. Again, no significant interaction effect was evident $(\beta=.12 ; \mathrm{t}=1.02 ; \mathrm{p}=.31)$.

Finally, a separate interaction term between maternal psychological control and positive parenting was added to the original first-order model; once more, no significant interaction effect was detected $(\beta=.12 ; \mathrm{t}=1.08 ; \mathrm{p}=.29)$.

\section{Discussion}

The current study set out to examine the main and interactive effects of three positive (i.e., parental involvement, positive parent-child communication, and positive parenting) and one negative (i.e., psychological control) parenting practice in relation to both children and mothers’ reports of child depressive symptoms. While no significant interactions between parenting practices were evident, results do suggest noteworthy patterns across reporters. Namely, children and mothers' reports of child depressive symptoms were unrelated, and differences emerged with respect to the relative importance both children and their mothers placed on individual parenting practices as they related to their respective reports of depressive symptoms.

Addressing the issue of multiple informants, the current study reified the all-to-common lack of correspondence between parents’ perceptions of their child’s depressive symptoms and 
the endorsement of symptomology by the child (De Los Reyes \& Kazdin, 2005). Understanding the importance attributed to unique parenting practices as they related to child depressive symptoms, therefore, must ultimately begin with the fact that children and their mothers reported largely discrepant levels of the outcome variable.

Indeed, differences across informants emerged, with regression analyses indicating that mothers endorsed a stronger association with parental involvement, and children endorsed a stronger association with psychological control. Consistent with hypotheses and previous research (Colder et al., 1997; Parent, Forehand, Dunbar, Watson, Reising, Seehuus, \& Compas, 2014), mothers endorsed a moderate negative association between their own active involvement in their child's life and their perceptions of their child's level of depressive symptomology, over and above the effect of other parenting practices. Thus, it may be that mothers attribute relatively greater importance to the degree to which they are actively engaging themselves in their child's life. It is not entirely surprising that children did not endorse such an association with their own symptoms. For one, past studies have demonstrated that, while parental involvement is generally associated with lower child depressive symptoms, if children perceive their parents to be overinvolved, the opposite is likelier to be true (Colder et al., 1997). Thus, parental involvement itself may not be a universally positive parenting practice per se (i.e., more is inherently better), but rather may operate on a continuum of effectiveness, making broad measures of parental involvement similar to the one used in the current study difficult to assess for optimum involvement. Furthermore, past research (e.g., Reynolds, 1992) has demonstrated that low correspondence between child and parent ratings of parental involvement is common, suggesting that these discrepancies may be related to a number of other factors, such as involvement expectation and parenting stress. 
Furthermore, parents and children's perceptions of parental influence often differ, and these differences can be related to family functioning. For example, McElhaney and colleagues (2008) found that differences between parent and child perceptions of parental influence on development were related to autonomy and control concerns, such that parents who endorsed greater perceptions of their own influence than their child exhibited more parental control and less engagement with their child in observed interactions. However, when children endorsed greater perceptions of parental influence, the opposite was true. Therefore, parents and children often have different perceptions of parental behavior, and it could be the case that increased parental reports of involvement are perceived by the child to be further instances of psychological control, which in our sample was uniquely predictive of children's reports of depressive symptoms. Consistent with past research (Barber, 1996; Barber et al., 2012), this finding would seem to indicate that psychological control has an overwhelmingly deleterious effect on children, regardless of the level of supportive parenting practices present.

Contrary to expectations and previous studies which found positive parenting to be negatively associated with child depressive symptoms (Bruce et al., 2006; Compas et al., 2010), no significant association between this parenting variable and either reporter's endorsement of child depressive symptoms was evident. Nevertheless, this finding is not altogether surprising. Past research has shown trends suggesting that as children age, parents' use of positive parenting practices tends to decline (e.g., Loeber et al., 2000). Accordingly, positive parenting may not be as influential for depressive symptoms as children progress to late childhood (and adolescence), an age range into which the current sample falls.

It should also be noted that in the current sample, both children and mothers' reports of child depressive symptoms were moderately negatively associated with positive parent-child 
communication. However, this association did not hold for either informant when controlling for the influence of other parenting practices. That is, while several noteworthy differences were evident with regard to parenting and reports of child depressive symptoms, communication seems to be salient to both children and their parents as a generally supportive parenting practice (Bayer et al., 2006). Nevertheless, communication may not be parenting behavior most strongly associated with child depressive symptoms. Psychological control and parental involvement appear to be stronger indicators of this association for the various informants.

Contrary to previous studies examining the co-occurrence of positive and negative parenting practices within the same parental source (e.g., Wimsatt et al., 2012), the expectation that high levels of psychological control in combination with high levels of supportive parenting would result in the highest levels of child-reported depressive symptoms was not supported by any of the findings. Taken together with the relation between psychological control alone and children's reports of their own depressive symptoms, this lack of an interaction may reflect a similar effect seen in the aforementioned studies examining competing parenting practices in different parental sources, such that the effects of negative parenting practices often outweigh the impact of the positive, particularly when the outcome is child depressive symptoms (Dallaire et al., 2006; Eisenberg et al., 2001; Harper et al., 2006).

Alternatively, whereas past studies demonstrating a similar effect to the hypothesized interaction (e.g., Turner \& Finkelhor, 1996, Wimsatt et al., 2012) focused on corporal punishment, which would be experienced subjectively as individual stressful life events, the current study focused on psychological control, which is more likely to be experienced as a chronic strain (Barber, 1996). This different experience of stress, particularly in the case of insidious psychological manipulation, may serve to diminish the stark inconsistency that a more 
concrete form of negative parenting (i.e., corporal punishment) might provide. Given that the hypothesized interaction was based on the theory that inconsistencies would be interpreted as parental rejection (Wimsatt et al., 2012), any mitigation of this inconsistency would serve to lessen the interactive effect. For example, Barber and colleagues (2002) argue that while psychological control is often experienced in the form of discrete behaviors, these behaviors may parallel consistent, key aspects of the overall parent-child relationship. In other words, parents that would not otherwise be considered physically abusive may still practice corporal punishment, but psychologically controlling behaviors are often encountered within parent-child relationships in which themes such as manipulation and guilt induction are common, thereby decreasing the apparent inconsistencies that these behaviors might display. Thus, with this particular form of stress, models such as the integrative model of parenting (Darling \& Steinberg, 1993; Lowe \& Dotterer, 2013), which stress interpretation of individual parenting practices in the context of the overall parent-child relationship, might offer additional aid in understanding its effect on child outcomes.

\section{Clinical Implications}

The current findings offer several implications with regard to working with children and families regarding issues of childhood depression. Given that maternal psychological control was uniquely associated with children's, but not mothers', reports of depressive symptoms, and that the opposite was found for parental involvement and mothers' reports, the current findings merit attention with regard to the role of parenting in the development of childhood depression. First, it is likely the case that parents and children are paying attention to different parental cues with regard to psychopathology (De Los Reyes \& Kazdin, 2005; Yeh \& Weisz, 2001). Additionally, their respective interpretations of the same behavior may differ as a function of other family 
dynamics (McElhaney, Porter, Thompson, \& Allen, 2008). Thus, it is critically important from an assessment perspective, to not only gather both parent and child reports of symptomology, but also to assess the differences perceived by parents and children in family functioning and how this relates to the child's depressive symptoms.

Second, contemporary efforts to demonstrate the harmful effects of corporal punishment have been successful to some effect in changing the cultural acceptance of these forms of discipline (Zolotor, Theodore, Runyan, Chang, \& Laskey, 2011). While many parents are choosing alternative disciplinary approaches to spanking, what is left unclear to many parents is the damage that negatively charged verbal punishments, such as psychological control, could have on their children. It may be the case that parents are unaware of their own psychologically controlling behavior, the effect it has on their child, or some degree of both. Thus, asking children and parents about psychologically controlling parental behavior, and focusing on how these are interpreted by the child, is another important part of the assessment process when dealing with child and adolescent depression. Possible targets for treatment may include educating families about healthy exercises of parental control and the need for developmentally appropriate child and adolescent autonomy.

\section{Limitations}

The current findings must be interpreted in consideration of some limitations. It should be noted that the current sample size had adequate power to detect medium to large, but not small, interaction effects (Aiken \& West, 1991). Previous studies examining similar interactions have yielded small (Turner \& Finkelhor, 1996) and medium to large effect sizes (Wimsatt et al., 2012). The sample utilized in the current study was a convenience sample and lacked sufficient diversity to be able to examine ethnicity as a moderator. Past studies have demonstrated 
differences across ethnic groups in rates of child depression (Garber et al., 2009), use of supportive parenting practices (McNeely \& Barber, 2010), and the effect of parental psychological control (Bean, Barber, \& Crane, 2006). Future studies would benefit by examining the hypothesized interactions within a range of ethnic demographics. Next, supportive parenting practices were assessed via mothers' self-report. While children's reports of their parents' behaviors would have provided an additional measure of validity, children, especially those with depression, may overrate their parents as being overly harsh or critical (Garber et al., 2009). Nevertheless, parents reporting on their own positive behavior still run the risk of a bias towards social desirability. Past studies comparing parental reports to documented parenting behavior (e.g., vaccination records or observed parenting interactions) have yielded mixed results (Morsbach \& Prinz, 2006), and that accuracy of parent-report can vary as a function of parental psychopathology (Parent et al., 2014). Indeed, each of the item-level mean scores for the three parenting practices assessed fell above a value of 4 (on a five-point Likert scale), indicating a restricted range of highly positive estimates of self-reported parenting. Also, it should be noted that significant first-order effects were only found within-raters, such that child-reported depressive symptoms were uniquely associated with child-reported parental psychological control, and mother-reported child depressive symptoms were uniquely associated with motherreported parental involvement. Thus, common method variance may account for some portion of these observed relationships. Future studies could increase the validity of assessing parental behavior through the incorporation of multiple reporters and/or methods (e.g., observation). The focus on mothers in the current study also limited the ability to examine the hypothesized interaction across parental sources. Past studies have shown that the rates and effects of psychological control can vary across parental source (i.e., father vs. mother; Bean et al., 2006). 
Future examinations of the co-occurrence of positive and negative parenting practices would benefit from looking at this effect in both parents. And finally, given that the current study was cross-sectional in its design, the directionality of parenting and child depression is assumed to be unidirectional (parenting impacts child depression). Past studies have yielded findings suggesting that this association may work in the opposite direction (e.g., Loukas, 2009; McLeod et al., 2007), or with a reciprocal effect (Soenens et al., 2008). For example, Loukas (2009) found that children's internalizing symptoms predicted changes in perceived maternal psychological control. Therefore, future studies utilizing a longitudinal design with multiple time points would allow for greater specificity of the relationship from parenting to childhood depression and vice versa. Conclusion

The nature of psychological control as a chronic parental source of stress (Barber, 1996) is clearly salient to children's reports of their own depressive symptoms. Parents, on the other hand, are likely to report a stronger link between their own involvement in their child's life and their perception of their child's depressive symptoms. The interaction of these sources of stress and support were not evident in the current study. Thus, more research is needed to fully elucidate the nature of how this insidious form of parental manipulation may likely interact with positive, supportive parenting practices in the development of child depressive symptoms. 
Table 1.

Descriptive Statistics for Relevant Study Variables

\begin{tabular}{lccc}
\hline & $\mathrm{M}$ & SD & Skew \\
\hline Age & 10.38 & 1.17 & .09 \\
Family Income & 58652.78 & 48352.44 & 1.10 \\
Psychological Control & 1.45 & .33 & .96 \\
Positive Communication & 4.13 & .30 & -.04 \\
Parental Involvement & 4.25 & .48 & -.03 \\
Positive Parenting & 4.09 & .45 & .17 \\
Depressive Symptoms - Child & .20 & .19 & 1.63 \\
Depressive Symptoms - Parent & 1.22 & .26 & 1.13 \\
\hline
\end{tabular}




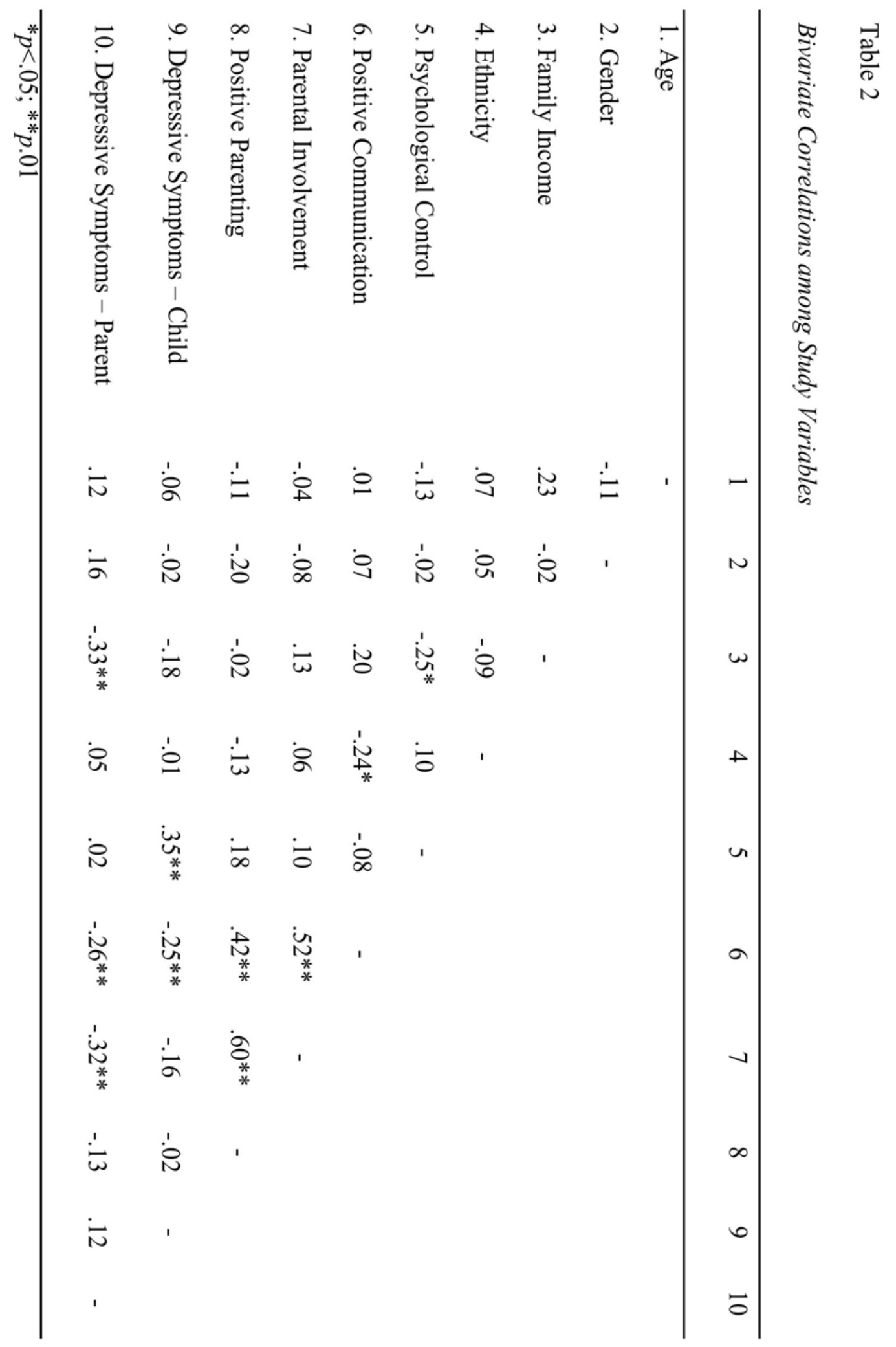


Table 3.

Children and Mothers' Reported Child Depressive Symptoms: First-Order Effects

\begin{tabular}{lcccc}
\hline \multirow{2}{*}{ Variable } & \multicolumn{2}{c}{ Child } & \multicolumn{2}{c}{ Mother } \\
\cline { 2 - 5 } & $\beta$ & SE & $\beta$ & SE \\
\hline Family Income & -.05 & .12 & $-.26^{*}$ & .11 \\
Maternal Psychological Control & $.34^{*}$ & .12 & .01 & .11 \\
Parent-Child & & & & .13 \\
Communication & -.14 & .14 & -.06 & .14 \\
Parental Involvement & & & & .13 \\
Positive Parenting & -.14 & .15 & $-.28^{*}$ & \\
\hline$* p<.05$ & .08 & .14 & .09 &
\end{tabular}




\section{References}

Achenbach, T. M., \& Rescorla, L. A. (2001). Manual for the ASEBA school-age forms \& profiles. Burlington, VT: University of Vermont, Research Center for Children, Youth, \& Families.

Aiken, L. S., \& West, S. G. (1991). Multiple Regression: Testing and Interpreting Interactions. Sage Publications, Inc, Thousand Oaks, CA. Retrieved from http://search.proquest.com/docview/618050774?accountid=14556

Barber, B. K. (1996). Parental psychological control: Revisiting a neglected construct. Child Development, 67(6), 3296-3319. Retrieved from http://search.proquest.com/docview/619053455?accountid=14556

Barber, B. K., \& Harmon, E. L. (2002). Violating the self: Parental psychological control of children and adolescents. (pp. 15-52) American Psychological Association, Washington, DC. doi:http://dx.doi.org/10.1037/10422-002

Barber, B. K., Stolz, H. E., \& Olsen, J. A. (2005). Parental support, psychological control, and behavioral control: Assessing relevance across time, culture, and method: I. Introduction. Monographs of the Society for Research in Child Development 70(4), 1-13.

Barber, B. K., Xia, M., Olsen, J. A., McNeely, C. A., \& Bose, K. (2012). Feeling disrespected by parents: Refining the measurement and understanding of psychological control. Journal of Adolescence, 35(2), 273-287.

Bayer, J. K., Sanson, A. V., \& Hemphill, S. A. (2006). Parent influences on early childhood internalizing difficulties. Journal of Applied Developmental Psychology, 27(6), 542-559. doi:http://dx.doi.org/10.1016/j.appdev.2006.08.002 
Bean, R. A., Barber, B. K., \& Crane, D. R. (2006). Parental support, behavioral control, and psychological control among African American youth: The relationships to academic grades, delinquency, and depression. Journal of Family Issues, 27(10), 1335-1355. doi:http://dx.doi.org/10.1177/0192513X06289649

Bidaut-Russell, M., Reich, W., Cottler, L. B., Robins, L. N., Compton, W. M., \& Mattison, R. E. (1995). The Diagnostic Interview Schedule for Children (PC-DISC v. 3.0): Parents and adolescents suggest reasons for expecting discrepant answers. Journal of Abnormal Child Psychology, 23(5), 641-659.

Boeldt, D. L., Rhee, S. H., DiLalla, L. F., Mullineaux, P. Y., Schulz-Heik, R. J., Corley, R. P., . . . Hewitt, J. K. (2012). The association between positive parenting and externalizing behaviour. Infant and Child Development, 21(1), 85-106. doi:http://dx.doi.org/10.1002/icd.764

Bradford, K., Barber, B. K., Olsen, J. A., Maughan, S. L., Erickson, L. D., Ward, D., \& Stolz, H. E. (2004). A multi-national study of interparental conflict, parenting, and adolescent functioning: South Africa, Bangladesh, China, India, Bosnia, Germany, Palestine, Colombia, and the United States. Marriage \& Family Review, 35(3-4), 107-137. doi:http://dx.doi.org/10.1300/J002v35n03_07

Brennan, P. A., Le Brocque, R., \& Hammen, C. (2003). Maternal depression, parent-child relationships, and resilient outcomes in adolescence. Journal of the American Academy of Child \& Adolescent Psychiatry, 42(12), 1469-1477. doi:http://dx.doi.org/10.1097/00004583-200312000-00014

Bruce, A. E., Cole, D. A., Dallaire, D. H., Jacquez, F. M., Pineda, A. Q., \& LaGrange, B. (2006). Relations of parenting and negative life events to cognitive diatheses for depression in 
children. Journal of Abnormal Child Psychology, 34(3), 321-333.

doi:http://dx.doi.org/10.1007/s10802-006-9019-x

Cicchetti, D., \& Toth, S. L. (1998). The development of depression in children and adolescents. American Psychologist, 53(2), 221-241. doi:http://dx.doi.org/10.1037/0003066X.53.2.221

Cohen, J. (1988). Statistical power analysis for the behavioral sciences (2nd ed.). Hillsdale, NJ: Erlbaum.

Colder, C. R., Lochman, J. E., \& Wells, K. C. (1997). The moderating effects of children's fear and activity level on relations between parenting practices and childhood symptomatology. Journal of Abnormal Child Psychology, 25(3), 251-263. Retrieved from http://search.proquest.com/docview/619079989?accountid=14556

Coln, K. L., Jordan, S. S., \& Mercer, S. H. (2013). A unified model exploring parenting practices as mediators of marital conflict and children's adjustment. Child Psychiatry and Human Development, 44(3), 419-429. doi:http://dx.doi.org/10.1007/s10578-012-0336-8

Compas, B. E., Champion, J. E., Forehand, R., Cole, D. A., Reeslund, K. L., Fear, J., . . Roberts, L. (2010). Coping and parenting: Mediators of 12-month outcomes of a family group cognitive-behavioral preventive intervention with families of depressed parents. Journal of Consulting and Clinical Psychology, 78(5), 623-634.

doi:http://dx.doi.org/10.1037/a0020459

Costello, E. J., Pine, D. S., Hammen, C., March, J. S., Plotsky, P. M., Weissman, M. M., . . Leckman, J. F. (2002). Development and natural history of mood disorders. Biological Psychiatry, 52(6), 529-542. doi:http://dx.doi.org/10.1016/S0006-3223(02)01372-0 
Dallaire, D. H., Pineda, A. Q., Cole, D. A., Ciesla, J. A., Jacquez, F., LaGrange, B., \& Bruce, A. E. (2006). Relation of positive and negative parenting to children's depressive symptoms. Journal of Clinical Child and Adolescent Psychology, 35(2), 313-322. doi:http://dx.doi.org/10.1207/s15374424jccp3502_15

Darling, N., \& Steinberg, L. (1993). Parenting style as context: An integrative model. Psychological Bulletin, 113(3), 487-496. doi:http://dx.doi.org/10.1037/00332909.113.3.487

DeLay, D., Hafen, C. A., Cunha, J. M., Weber, L. N. D., \& Laursen, B. (2013). Perceptions of parental support buffer against depression for Brazilian youth with interpersonal difficulties. International Journal of Behavioral Development, 37(1), 29-34. doi:http://dx.doi.org/10.1177/0165025412454031

De Los Reyes, A. \& Kazdin, A. E. (2005). Informant discrepancies in the assessment of childhood psychopathology: A critical review, theoretical framework, and recommendations for further study. Psychological Bulletin, 131(4), 483-509. doi:http://dx.doi.org/10.1037/0033-2909.131.4.483

Drake, K. L., \& Ginsburg, G. S. (2012). Family factors in the development, treatment, and prevention of childhood anxiety disorders. Clinical Child and Family Psychology Review, 15(2), 144-162. doi:http://dx.doi.org/10.1007/s10567-011-0109-0

Duchesne, S., \& Ratelle, C. (2010). Parental behaviors and adolescents' achievement goals at the beginning of middle school: Emotional problems as potential mediators. Journal of Educational Psychology, 102(2), 497-507. doi:http://dx.doi.org/10.1037/a0019320

Eisenberg, N., Gershoff, E. T., Fabes, R. A., Shepard, S. A., Cumberland, A. J., Losoya, S. H., . . Murphy, B. C. (2001). Mother's emotional expressivity and children's behavior 
problems and social competence: Mediation through children's regulation. Developmental Psychology, 37(4), 475-490.

El Nokali, N. E, Bachman, H. J. \& Vortruba-Drzal, E. (2010). Parent involvement and children’s academic and social development in elementary school. Child Development, 81, 9881005. doi: 10.1111/j.1467-8624.2010.01447.x

Fan, X., \& Chen, M. (2001). Parental involvement and students' academic achievement: A metaanalysis. Educational Psychology Review, 13, 1-22.

Garber, J., Gallerani, C. M., \& Frankel, S. A. (2009). Depression in children. Guilford Press, New York, NY. Retrieved from http://search.proquest.com/docview/621690001?accountid=14556

Gaté, M. A., Watkins, E. R., Simmons, J. G., Byrne, M. L., Schwartz, O. S., Whittle, S., . . Allen, N. B. (2013). Maternal parenting behaviors and adolescent depression: The mediating role of rumination. Journal of Clinical Child and Adolescent Psychology, 42(3), 348-357. doi:http://dx.doi.org/10.1080/15374416.2012.755927

Harper, F. W. K., Brown, A. M., Arias, I., \& Brody, G. (2006). Corporal punishment and kids: How do parent support and gender influence child adjustment? Journal of Family Violence, 21(3), 197-207. doi:http://dx.doi.org/10.1007/s10896-006-9018-2

Harrington, R., Rutter, M., \& Fombonne, E. (1996). Developmental pathways in depression: Multiple meanings, antecedents, and endpoints. Development and Psychopathology, 8, 601-616.

Healey, D. M., Flory, J. D., Miller, C. J., \& Halperin, J. M. (2011). Maternal positive parenting style is associated with better functioning in hyperactive/inattentive preschool 
children. Infant and Child Development, 20(2), 148-161.

doi:http://dx.doi.org/10.1002/icd.682

Kolko, D. J., \& Kazdin, A. E. (1993). Emotional/behavioral problems in clinic and nonclinic children: Correspondence among child, parent and teacher reports. Child Psychology \& Psychiatry \& Allied Disciplines, 34(6), 991-1006. Retrieved from http://search.proquest.com/docview/618427284?accountid=14556

Kovacs, M. (1992). Children’s Depression Inventory. Toronto: Multi-Health Systems.

Kovacs, M. (1983). Children’s Depression Inventory. Unpublished Manuscript, University of Pittsburg, Pennsylvania.

Lewinsohn, P. M., Hops, H., Roberts, R. E., Seeley, J. R., \& Andrews, J. A. (1993). Adolescent psychopathology: I. Prevalence and incidence of depression and other DSM-III—R disorders in high school students. Journal of Abnormal Psychology, 102(1), 133-144. doi:http://dx.doi.org/10.1037/0021-843X.102.1.133

Lima, N. N. R., do Nascimento, V. B., de Carvalho, S. M. F., de Abreu, L. C., Neto, M. L. R., Brasil, A. Q., ... \& Reis, A. O. A. (2013). Childhood depression: a systematic review. Neuropsychiatric disease and treatment, 9, 1417.

Loeber, R., Farrington, D. P., Stouthamer-Loeber, M. \& Van Kammen, W. B. (1998). Antisocial behavior and mental health problems: Explanatory factors in childhood and adolescence. Mahwah, NJ: Erlbaum.

Loukas, A. (2009). Examining temporal associations between perceived maternal psychological control and early adolescent internalizing problems. Journal of abnormal child psychology, 37(8), 1113-1122. 
Lowe, K., \& Dotterer, A. M. (2013). Parental monitoring, parental warmth, and minority youths’ academic outcomes: Exploring the integrative model of parenting. Journal of Youth and Adolescence, 42(9), 1413-1425. doi:http://dx.doi.org/10.1007/s10964-013-9934-4

Lyneham, H. J., \& Rapee, R. M. (2006). Evaluation of therapist-supported parent-implemented CBT for anxiety disorders in rural children. Behaviour research and therapy, 44(9), $1287-1300$.

Mandara, J., \& Pikes, C. L. (2008). Guilt trips and love withdrawal: Does mothers' use of psychological control predict depressive symptoms among african american adolescents? Family Relations: An Interdisciplinary Journal of Applied Family Studies,57(5), 602-612. doi:http://dx.doi.org/10.1111/j.1741-3729.2008.00526.x

McElhaney, K. B., Porter, M. R., Thompson, L. W., \& Allen, J. P. (2008). Apples and oranges: Divergent meanings of parents' and adolescents' perceptions of parental influence. The Journal of early adolescence.

McLeod, B. D., Weisz, J. R., \& Wood, J. J. (2007). Examining the association between parenting and childhood depression: A meta-analysis. Clinical psychology review, 27(8), 986-1003.

Morsbach, S. K., \& Prinz, R. J. (2006). Understanding and improving the validity of self-report of parenting. Clinical Child and Family Psychology Review, 9(1), 1-21.

1.

Muris, P., Meesters, C., Schouten, E., \& Hoge, E. (2004). Effects of perceived control on the relationship between perceived parental rearing behaviors and symptoms of anxiety and depression in nonclinical preadolescents. Journal of Youth and Adolescence, 33(1), 51-58. doi:http://dx.doi.org/10.1023/A:1027334314021 
Murray, K. W., Dwyer, K. M., Rubin, K. H., Knighton-Wisor, S., \& Booth-LaForce, C. (2013). Parent-child relationships, parental psychological control, and aggression: Maternal and paternal relationships. Journal of Youth and Adolescence. doi:http://dx.doi.org/10.1007/s10964-013-0019-1

Myers, K., \& Winters, N.C. (2002). Ten-year review of rating scales II: Scales for internalizing disorders. Journal of American Academy of Child and Adolescent Psychiatry, 41, 634659.

Nurcombe, B., Seifer, R., Scioli, A., Tramuntana, M., Lexington, W., \& Beauchesne, H. (1989). Is major depressive disorder in adolescence a distinct diagnostic entity. Journal of the American Academy of Child and Adolescent Psychiatry, 28, 333-342.

Pardini, D.A., Fite, P., \& Burke, J.D. (2008). Bidirectional asso- ciations between parenting practices and conduct problems in boys from childhood to adolescence: The moderating effect of age and African-American ethnicity. Journal of Abnormal Child Psychology, 36, 647-662.

Parent, J., Forehand, R., Dunbar, J. P., Watson, K. H., Reising, M. M., Seehuus, M., \& Compas, B. E. (2014). Parent and adolescent reports of parenting when a parent has a history of depression: Associations with observations of parenting. Journal of abnormal child psychology, 42(2), 173-183.

Pearlin, L. I., Menaghan, E. G., Lieberman, M. A., \& Mullan, J. T. (1981). The stress process. Journal of Health and Social Behavior, 22(4), 337-356. Retrieved from http://search.proquest.com/docview/616629646?accountid=14556 
Pearson, J., Muller, C., \& Frisco, M. L. (2006). Parental involvement, family structure, and adolescent sexual decision making. Sociological Perspectives, 49(1), 67-90. doi:http://dx.doi.org/10.1525/sop.2006.49.1.67

Plunkett, S. W., Henry, C. S., Robinson, L. C., Behnke, A., \& Falcon,Pedro C., I.,II. (2007). Adolescent perceptions of parental behaviors, adolescent self-esteem, and adolescent depressed mood. Journal of Child and Family Studies, 16(6), 760-772. doi:http://dx.doi.org/10.1007/s10826-006-9123-0

Reynolds, A. J. (1992). Comparing measures of parental involvement and their effects on academic achievement. Early Childhood Research Quarterly, 7(3), 441-462.

Rohner, R. P., Bourque, S. L., \& Elordi, C. A. (1996). Children's perceptions of corporal punishment, caretaker acceptance, and psychological adjustment in a poor, biracial southern community. Journal of Marriage and the Family, 58(4), 842-852. Retrieved from http://search.proquest.com/docview/619195201?accountid=14556

Schaefer, E. S. (1965). Children's reports of parental behavior: An inventory. Child Development, 36(2), 413-424. Retrieved from http://search.proquest.com/docview/615448073?accountid=14556

Soenens, B., Luyckx, K., Vansteenkiste, M., Duriez, B., \& Goossens, L. (2008). Clarifying the link between parental psychological control and adolescents' depressive symptoms: Reciprocal versus unidirectional models. Merrill-Palmer Quarterly, 54(4), 411-444. doi:http://dx.doi.org/10.1353/mpq.0.0005

Stouthamer-Loeber, M., Loeber, R., Wei, E., Farrington, D. P., \& Wikström, P. H. (2002). Risk and promotive effects in the explanation of persistent serious delinquency in 
boys. Journal of Consulting and Clinical Psychology, 70(1), 111-123.

doi:http://dx.doi.org/10.1037/0022-006X.70.1.111

Taylor, R. D., Lopez, E. I., Budescu, M., \& McGill, R. K. (2012). Parenting practices and adolescent internalizing and externalizing problems: Moderating effects of socially demanding kin relations. Journal of Child and Family Studies, 21(3), 474-485.

Tolou-Shams, M., Paikoff, R., McKirnan, D. J., \& Holmbeck, G. N. (2007). Mental health and HIV risk among african american adolescents: The role of parenting. Social Work in Mental Health, 5(1-2), 27-58. doi:http://dx.doi.org/10.1300/J200v05n01_02

Treutler, C. M., \& Epkins, C. C. (2003). Are discrepancies among child, mother, and father reports on children's behavior related to parents' psychological symptoms and aspects of parent-child relationships? Journal of Abnormal Child Psychology, 31(1), 13-27. doi:http://dx.doi.org/10.1023/A:1021765114434

Turner, H. A., \& Finkelhor, D. (1996). Corporal punishment as a stressor among youth. Journal of Marriage and the Family, 58(1), 155-166. Retrieved from http://search.proquest.com/docview/619190762?accountid=14556

Vazsonyi, A. T., \& Belliston, L. M. (2006). The cultural and developmental significance of parenting processes in adolescent anxiety and depression symptoms. Journal of Youth and Adolescence, 35(4), 491-505. doi:http://dx.doi.org/10.1007/s10964-006-9064-3

Wimsatt, A. R., Fite, P. J., Grassetti, S. N., \& Rathert, J. L. (2013). Positive communication moderates the relationship between corporal punishment and child depressive symptoms. Child and Adolescent Mental Health, 18(4), 225-230. 
Yeh, M., \& Weisz, J. R. (2001). Why are we here at the clinic? Parent-child (dis) agreement on referral problems at outpatient treatment entry. Journal of Consulting and Clinical Psychology, 69(6), 1018.

Yu, S., Clemens, R., Yang, H., Li, X., Stanton, B., Deveaux, L., . . . Harris, C. (2006). Youth and parental perceptions of parental monitoring and parent-adolescent communication, youth depression, and youth risk behaviors. Social Behavior and Personality, 34(10), 12971310. doi:http://dx.doi.org/10.2224/sbp.2006.34.10.1297

Zolotor, A. J., Theodore, A. D., Runyan, D. K., Chang, J. J., \& Laskey, A. L. (2011). Corporal punishment and physical abuse: population-based trends for three-to-11-year-old children in the United States. Child abuse review, 20(1), 57-66. 


\section{RESEARCH PARTICIPANTS NEEDED UT Child Behavior Lab}

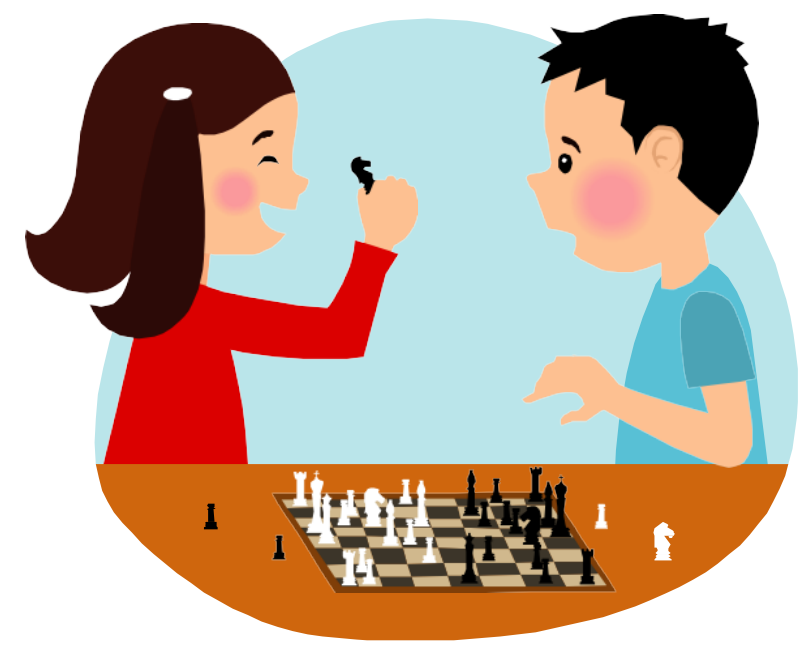

Do you have a child ranging from 9 to 12 years of age?

We are looking for children with and without behavior problems to participate in a study that examines the development of problem behavior. You and your child will be asked to answer questions, and

your child will play two computer games. The study will take approximately 2 hours. You will be compensated $\$ 45$ for your time.

\section{For more information \\ Call (865) 974-5306}

\begin{tabular}{|c|c|c|c|c|c|c|c|c|c|c|c|}
\hline 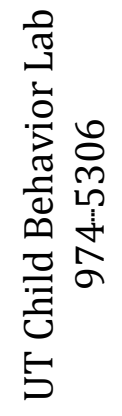 & 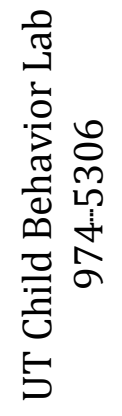 & 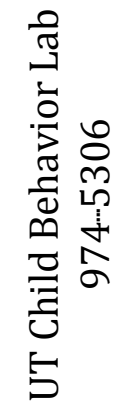 & 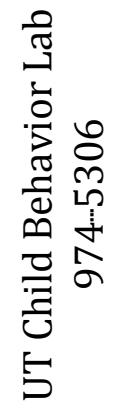 & 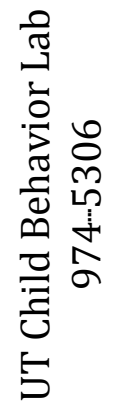 & 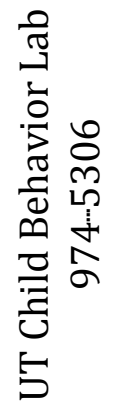 & 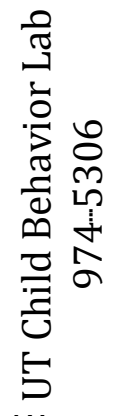 & 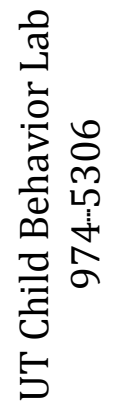 & 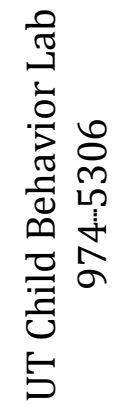 & 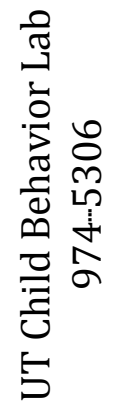 & 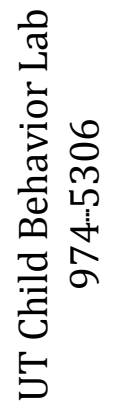 & 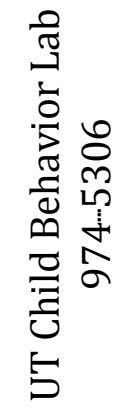 \\
\hline
\end{tabular}

